\title{
Mechanism underlying renal failure caused by pathogenic Candida albicans infection
}

\author{
JAE-CHEN SHIN ${ }^{1 *}$, YOUNG-JOO JEON ${ }^{2 *}$, SEON-MIN PARK ${ }^{1}$, \\ KANG SEOK SEO ${ }^{3}$, JUNG-HYUN SHIM ${ }^{4}$ and JUNG-IL CHAE ${ }^{2}$ \\ ${ }^{1}$ Pohang Center for Evaluation of Biomaterials, Pohang, Gyeongbuk; ${ }^{2}$ Department of Oral Pharmacology, \\ School of Dentistry and Institute of Oral Bioscience, BK21 plus, Chonbuk National University, Jeonju; \\ ${ }^{3}$ Department of Animal Science and Technology, Sunchon National University, Suncheon; \\ ${ }^{4}$ Natural Medicine Research Institute, Department of Pharmacy, College of Pharmacy, \\ Mokpo National University, Muan, Jeonnam, Republic of Korea
}

Received October 14, 2014; Accepted October 29, 2014

DOI: $10.3892 /$ br.2014.393

\begin{abstract}
Candida albicans (C. albicans) is an opportunistic fungal pathogen that commonly causes nosocomial infections. Systemic candidiasis is encountered with increasing frequency in immunocompromised hosts, leading to renal failure that results in severe morbidity and mortality. The present study investigated the mechanisms underlying kidney susceptibility following infection with several $C$. albicans strains, such as B311 and SC5314. Fungal growth of the highly virulent SC5314 strain was $10^{3}$-fold higher compared to the nonpathogenic B311 strain in the kidneys. An intravenous challenge of SC5314 in mice, elevated blood urea nitrogen (BUN) and creatine levels, which resulted in mortality at 8 or 35 days after infection in a dose- and time-dependent manner, whereas all the B311-infected mice had BUN and creatinine levels in the normal range and survived. Whether virulent $C$. albicans may escape clearance by activating signaling pathways that lead to the production of pro-inflammatory cytokines, such as tumor necrosis factor (TNF)- $\alpha$ and interleukin (IL)-1 $\beta$, was investigated. B311 infections significantly elevated $T N F-\alpha$ and $I L-1 \beta$ mRNA expression in the kidneys, whereas the expression in SC5314-infected mice remained unchanged. Furthermore, B311 infection significantly elevated the plasma levels of TNF- $\alpha$ and IL-1 $\beta$. These results indicated that the less virulent strains of $C$. albicans induced
\end{abstract}

Correspondence to: Professor Jung-Il Chae, Department of Oral Pharmacology, School of Dentistry, BK21 plus, Chonbuk National University, Jeonju 651-756, Republic of Korea

E-mail: jichae@jbnu.ac.kr

Professor Jung-Hyun Shim, Department of Pharmacy, College of Pharmacy, Mokpo National University, 1666 Youngsan-ro, Muan-gun, Jeonnam 534-729, Republic of Korea

E-mail: s1004jh@gmail.com

"Contributed equally

Key words: Candida albicans, B311, SC5314, tumor necrosis factor- $\alpha$, interleukin- $1 \beta$ pro-inflammatory cytokines in mice. These results determined that an impairment of the protective mechanisms occurred in the kidneys with virulent $C$. albicans infection.

\section{Introduction}

Candida albicans (C. albicans) is an infective diploid with a filamentous or yeast-like morphology that causes intradermal or subcutaneous candidiasis, vaginitis and systemic mycosis by infecting the skin or mucous membranes (1). C. albicans infection does not cause symptoms in healthy individuals but results in mortality in immunocompromised individuals, such as those with acquired immune deficiency syndrome or cancer, organ or bone marrow transplant patients, or those that have been taking immunosuppressant drugs for an extended period of time (2). The pathogenicity of $C$. albicans and the host defense mechanisms involved have been widely studied in mouse models to develop effective antifungal agents (3-8). Animal studies have focused on survival factors, however the exact mechanisms underlying mortality in $C$. albicans-infected mice remain unknown.

The identification of pathogenic factors mediating C.albicans infection will aid in elucidating the mechanisms that lead to fatality. The first biological process of $C$. albicans infection is the attachment to the mucosa or other regions in a living organism. Phenotypic switching from yeast to pseudohyphae or hyphae is required for tissue infiltration, processes mediated by extracellular proteinases and extracellular phospholipases (9-12). However, the impact of pathogenic $C$. albicans infection on the immune response of the host is unknown. The dominant theory is that the major cause of mortality by C. albicans infection is disseminated candidiasis by fungal growth within the kidney and consequent kidney damage (13). Elevations in blood urea nitrogen (BUN) and serum creatinine are frequently evaluated to measure the degree of kidney damage, such as renal failure by infection or other factors (14). Urea is the primary metabolite derived from dietary and tissue protein, while creatinine is the catabolic product of muscle creatine.

Chronic kidney disease is associated with a variety of cytokines, including the pro-inflammatory cytokines, interleukin 
(IL)-1 $\beta$ and tumor necrosis factor (TNF)- $\alpha(15,16)$. Cytokines have pleiotropic actions on target tissues with autocrine and apocrine physiological effects. The pro-inflammatory cytokines are controlled by anti-inflammatory cytokines, such as IL-10, and have important roles in the regulation of immune responses due to infection. The C.albicans strain, SC5314, has been widely applied in previous genetic and laboratory studies. In vivo, SC5314 is highly pathogenic due to rapid penetration, while another $C$. albicans strain, B311, forms pseudohyphae with a lower pathogenicity than SC5314 $(17,18)$. The study of fungal keratitis using $C$. albicans strains is ongoing, but the role of kidney damage during treatment and the mechanisms underlying $C$. albicans pathogenicity have not been widely reported $(19,20)$. Therefore, the present study assessed kidney damage originating from systemic administration of pathogenic or nonpathogenic $C$. albicans strains in mice.

\section{Materials and methods}

C. albicans. C. albicans B311 (nonpathogenic) and SC5314 (pathogenic) were kindly provided by Professor Kenji Tanaka (University of Nagoya, Nagoya, Japan). C.albicans were grown to a stationary phase at $30^{\circ} \mathrm{C}$ with slight agitation in Sabouraud dextrose broth (BD Microbiology Systems, Sparks, MD, USA). After $24 \mathrm{~h}$ culture, the cells were harvested by centrifugation $(2,000 \mathrm{x} \mathrm{g})$, washed twice in phosphate-buffered saline (PBS), diluted to the desired density and injected intravenously (i.v.) in a $0.1 \mathrm{ml}$ volume.

Animals. Specific pathogen-free female BALB/c mice were obtained from the Pohang University of Science and Technology POSTECH IACUC approval no. 2010-03-0008 (Kyeongbuk, Korea). The mice were housed in a laminar flow cabinet and maintained on standard laboratory chow ad libitum. All mice were used at the age of 8-10 weeks.

Quantification of C. albicans. Kidneys from individual mice were removed aseptically and homogenized with $0.2 \mathrm{ml}$ PBS. The number of viable colony-forming units in the specimens was determined by dilution plating on Sabouraud dextrose agar (BD Diagnostic Systems).

Quantitative polymerase chain reaction (qPCR). Total cellular RNA was isolated using the single-step method and TRIzol reagent (Sigma, St. Louis, MO, USA). Reverse transcription was performed with $2 \mu \mathrm{g}$ total RNA in a $10 \mu \mathrm{l}$ reaction mixture (Promega, Madison, WI, USA) containing oligo (dT)15 and avian myeloblastosis virus reverse transcriptase. A PCR reaction mixture containing SYBR-Green (SYBR premix ExTaq; Takara Shuzo, Kyoto, Japan) in a final $20 \mu$ l volume reaction mix, containing $10 \mu \mathrm{l}$ PCR mixture, $2 \mu \mathrm{l}$ specific primers $(10 \mathrm{pM}), 7 \mu \mathrm{l}$ nuclease-free water and $1 \mu \mathrm{l}$ cDNA. Amplification was performed with a Rotor-Gene 3000 (Corbett Research Pty Ltd, Sydney, Australia) programmed at $95^{\circ} \mathrm{C}$ for $15 \mathrm{~min}$ followed by 50 cycles of $95^{\circ} \mathrm{C}$ for $10 \mathrm{sec}, 55 \sim 61^{\circ} \mathrm{C}$ for $20 \mathrm{sec}$ and $72^{\circ} \mathrm{C}$ for $30 \mathrm{sec}$. Data were collected and analyzed with the Corbett Research Software (version 6.0). The comparative critical threshold values from four measurements were used to calculate the gene expression, with normalization to $\beta$-actin as an internal control. The primers used were as follows: $T N F-\alpha$,
5'-CTGTAGCCCACGTCGTAGC-3' and 5'-TTGAGATCCA TGCCGTTG-3; and $\beta$-actin, 5'-CTGAAGTCACCCATTGA ACATGGC-3' and 5'-CAGAGAGTAATCTCCTTCTGCAT-3'.

Whole-blood chemistry parameters. Whole-blood chemistry parameters were obtained using EC $8+$ and Crea cartridges (Heska Corporation, Loveland, CO, USA). The EC $8+$ cartridges measure BUN and the Crea cartridges measure creatinine. Mice were anticoagulated with heparin (100 units administered intraperitoneally; Sigma) 5-15 min before euthanasia to allow sufficient blood to be harvested and prevent clotting in the cartridges. Whole blood was aspirated into a heparinized 25 -gauge syringe and $\sim 100 \mu \mathrm{l}$ was aliquoted into the appropriate cartridges, in accordance with the manufacturer's instructions.

Statistical analyses. Data were presented as the mean \pm standard error. Statistical significance was determined using the analysis of variance test (StatView; Abacus Concepts Inc., Berkeley, CA, USA). All the experiments were performed at least three times and $\mathrm{P}<0.05$ was considered to indicate a statistically significant difference.

\section{Results}

Response to C. albicans infection in mice. The weak pathogenic strain, B311, or the potent pathogenic strain, SC5314, were injected into the tail vein of mice at a concentration of $5 \times 10^{5}$, to assess kidney damage caused by pathogenic C. albicans infection. The mice were sacrificed after $48 \mathrm{~h}$ and fungal growth in the kidney was measured. The fungal growth of the virulent SC5314 was $\sim 10^{3}$ higher compared to the nonpathogenic B311 strain (Fig. 1A). Additionally, mice infected with B311 survived 35 days, whereas all the mice infected with $2 \times 10^{6}$ SC5314 succumbed 8 days after infection and those infected with $1 \times 10^{6}$ SC5314 survived 35 days (Fig. 1B). These results indicated that host defense mechanisms specific to pathogenic C. albicans are not present in the kidney. Therefore, increased fungal growth in the kidney may influence the survival rate.

Pro-inflammatory cytokines are highly associated with the C. albicans infection response in mice. The renal pro-inflammatory cytokines, such as TNF- $\alpha$ and IL-1 $\beta$, which play major roles in protection from infectious microbes in B311- and SC5314-infected mice were comparatively analyzed. RNA was extracted from mice following infection and qPCR was performed to analyze $T N F-\alpha$ and $I L-1 \beta$. TNF- $\alpha$ mRNA increased significantly at $2 \mathrm{~h}$, followed by a decrease at $8 \mathrm{~h}$ in B311-infected mice. However, SC5314-infected mice showed no significant increase in $T N F-\alpha$ expression (Fig. $2 \mathrm{~A}$ ). $I L-1 \beta$ mRNA significantly increased in B311-infected mice at 4 and $8 \mathrm{~h}$, while there was no significant increase in $I L-1 \beta$ expression in SC5314-infected mice (Fig. 2B). Enzyme-linked immunosorbent assay (ELISA) analysis was also performed to determine the protein expression of TNF- $\alpha$ and IL- $1 \beta$ in mice infected with B311 or SC5314 C. albicans strains, and confirmed the mRNA expression studies described above. TNF- $\alpha$ protein expression was increased in mouse kidneys in 1-2 $\mathrm{h}$, followed by a decrease at $4 \mathrm{~h}$; while IL-1 $\beta$ increased after 4 and $8 \mathrm{~h}$. By contrast, SC5314-infected mice showed no significant 
A

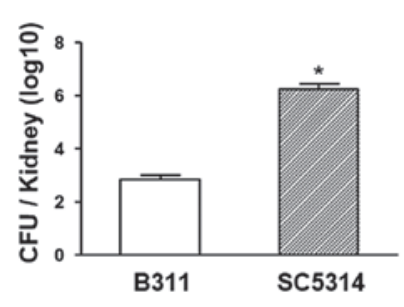

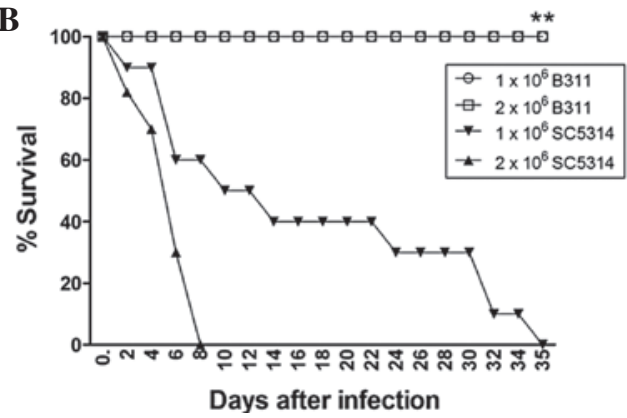

Figure 1. Comparison of fungal growth and survival rate between B311 and SC5314 Candida albicans (C. albicans) strains. (A) Mice were infected intravenously (i.v.) with $5 \times 10^{5}$ B 311 or SC5314 for determining the fungal growth ( $\mathrm{n}=5$ animals per group) in kidneys ( $\mathrm{n}=5$ animals per group). "P<0.05 compared to the B311-infected group. (B) Mice were infected i.v. with $1 \times 10^{6}$ and $2 \times 10^{6}$ C. albicans for determining the survival rate of mice ( $\mathrm{n}=10$ animals per group). ${ }^{*} \mathrm{P}<0.05$ compared to the SC5314-infected group. Values are expressed as means \pm standard error. CFU, colony-forming unit.
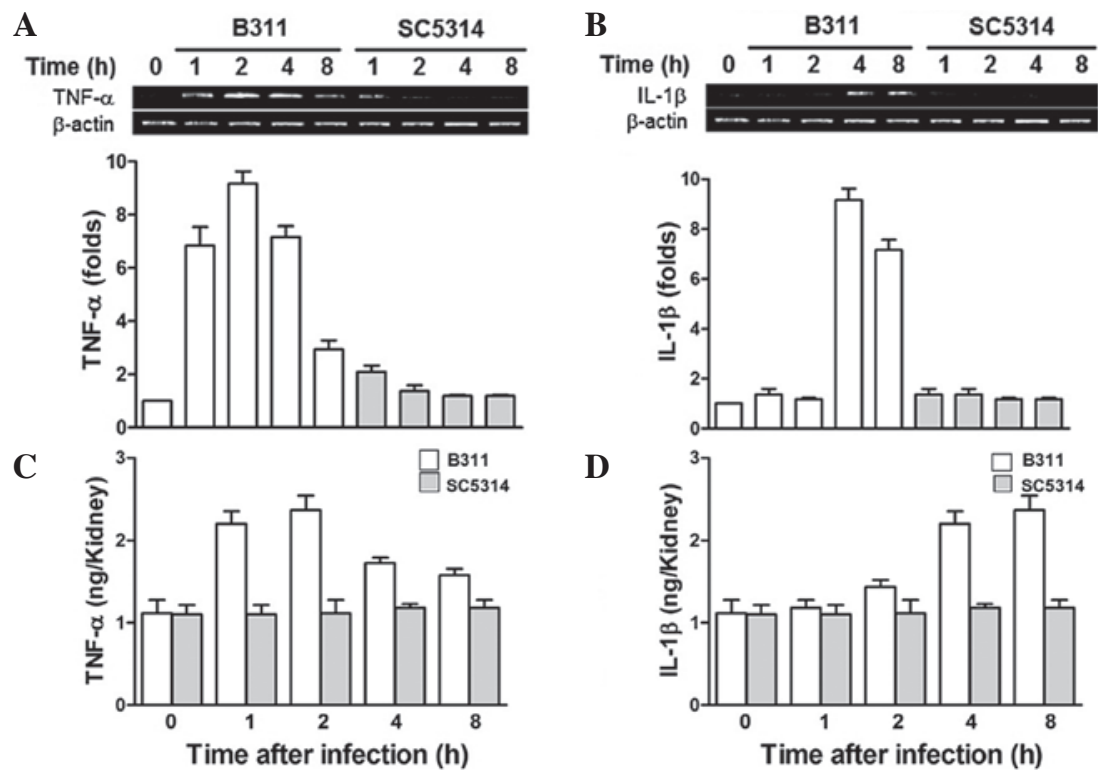

Figure 2. Tumor necrosis factor (TNF)- $\alpha$ and interleukin (IL)-1 $\beta$ expression of Candida albicans-infected mice. Mice were infected with $1 \times 10^{6}$ B311 or SC5413 and sacrificed at the indicated times. (A) $T N F-\alpha$ and (B) $I L-1 \beta$ mRNA levels were determined by quantification polymerase chain reaction. The standard error of the mean is indicated for each sample. (C) TNF- $\alpha$ and (D) IL-1 $\beta$ cytokine levels were determined by enzyme-linked immunosorbent assay. The standard error of the mean is indicated for each sample.
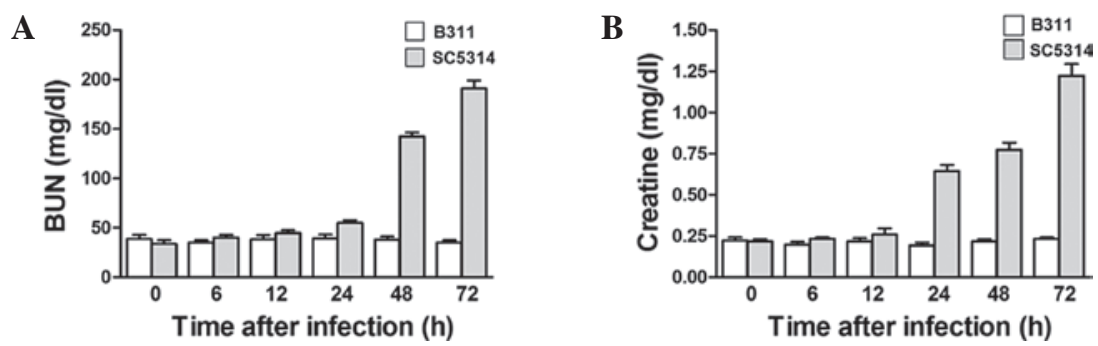

Figure 3. Blood urea nitrogen (BUN) and creatine levels were increased in mouse serum by virulent Candida albicans (C. albicans) (SC5314) infection. (A) In contrast to the acute rise in BUN observed in virulent $C$. albicans-infected mice, BUN levels were completely unchanged in low-virulent $C$. albicans (B311)-infected mice. (B) Creatine levels were completely unchanged in low-virulent $C$. albicans (B311)-infected mice in contrast to the acute rise in creatine observed in virulent $C$. albicans-infected mice.

TNF- $\alpha$ and IL-1 $\beta$ protein expression (Fig. $2 \mathrm{C}$ and D). These results indicated that early immune responses mediated by pro-inflammatory cytokines, such as TNF- $\alpha$ and IL-1 $\beta$, attenuated the pathogenicity of $C$. albicans, since the highly pathogenic C. albicans strain, SC5314, suppressed early immune responses.
Virulent C. albicans infection is affected with renal failure. BUN and creatine measurements were used to diagnose renal dysfunction. Creatine is a ubiquitous enzyme that exists in the heart, musculoskeletal system, brain and kidney and circulates in response to tissue destruction. Mice infected with the highly pathogenic SC5314 strain showed 
a significant increase in blood BUN and creatine at 48 and $72 \mathrm{~h}$ after infection, while infection with nonpathogenic B311 showed no significant changes (Fig. 3A and B). These results indicated that SC5314-infected mice developed strong pathogenicity one day after infection, coupled to severe renal failure.

\section{Discussion}

C. albicans is a representative infectious pathogen of the skin or mucosal layers in immunocompromised patients $(1,2)$. However, a comprehensive study of the physiological responses of mice with candidiasis or other fungal infection has not been reported. Although $C$. albicans-mediated renal failure is an important hemodynamic or chemical parameter in mouse models, the mechanisms underlying candidiasis-mediated fatality have not been clearly elucidated (21).

Kidney damage was assessed in mice originating from systemic administration using pathogenic or nonpathogenic C. albicans strains. Mice were intravenously infected with the highly pathogenic $C$. albicans strain, SC5314, or the nonpathogenic $C$. albicans strain, B311. Fungal growth in the kidney was verified to analyze the association between fungal growth-mediated renal failure and lethality. SC5314-infected mice showed more severe fungal growth compared to B311-infected mice. The survival rate of SC5314-infected mice was also significantly reduced compared to B311-infected mice. These data indicated that lethality in mice was governed by the level of renal failure post- $C$. albicans infection, and that the pathogenicity of $C$. albicans was determined by the level of renal failure.

Pro-inflammatory cytokines, such as TNF- $\alpha$ and IL-1 $\beta$, which play major roles in pathogenic infection were analyzed by qPCR and ELISA, to elucidate the underlying factors of C. albicans pathogenicity. TNF- $\alpha$ and IL- $1 \beta$ were significantly increased in B311-, but not SC5314-, infected mice suggesting that the pathogenicity of $C$. albicans was determined by early pro-inflammatory cytokine actions.

Chronic renal dysfunction or failure was further assessed by measuring urea and creatine blood concentrations. SC5314-infected mice showed an increase in blood urea and creatine two and one day post-infection, respectively. B311-infected mice showed no significant increase in the levels of urea and creatine in accordance with a previous analysis of mice lethality (22).

In conclusion, systemic infection of $C$. albicans induced severe renal failure, as determined by blood BUN and creatine levels. The working model demonstrated that severe renal failure plays a major role in lethality of $C$. albicans-infected mice. Early expression of renal pro-inflammatory cytokines is also a major determinant of pathogenicity. These results increase the understanding of $C$. albicans pathogenicity together with the underlying mechanisms of morbidity and mortality in C. albicans infection.

\section{Acknowledgements}

This study was supported by a grant from the Next-Generation BioGreen 21 Program (no. PJ00811604) and the Rural Development Administration, Republic of Korea.

\section{References}

1. Kim J and Sudbery P: Candida albicans, a major human fungal pathogen. J Microbiol 49: 171-177, 2011.

2. Arulanantham K, Dwyer JM and Genel M: Evidence for defective immunoregulation in the syndrome of familial candidiasis endocrinopathy. N Engl J Med 300: 164-168, 1979.

3. Netea MG, Van Der Graaf CA, Vonk AG, Verschueren I, Van Der Meer JW and Kullberg BJ: The role of toll-like receptor (TLR) 2 and TLR4 in the host defense against disseminated candidiasis. J Infect Dis 185: 1483-1489, 2002.

4. Vonk AG, Netea MG, van Krieken JH, van der Meer JW and Kullberg BJ: Delayed clearance of intraabdominal abscesses caused by Candida albicans in tumor necrosis factor-alpha- and lymphotoxin-alpha-deficient mice. J Infect Dis 186: 1815-1822, 2002.

5. Davis D, Edwards JE Jr, Mitchell AP and Ibrahim AS: Candida albicans RIM101 $\mathrm{pH}$ response pathway is required for host-pathogen interactions. Infect Immun 68: 5953-5959, 2000.

6. Brieland J, Essig D, Jackson C, et al: Comparison of pathogenesis and host immune responses to Candida glabrata and Candida albicans in systemically infected immunocompetent mice. Infect Immun 69: 5046-5055, 2001.

7. Clemons KV and Stevens DA: Efficacy of the partricin derivative SPA-S-753 against systemic murine candidosis. J Antimicrob Chemother 47: 183-186, 2001.

8. Graybill JR, Bocanegra R, Najvar LK, Hernandez S and Larsen RA: Addition of caspofungin to fluconazole does not improve outcome in murine candidiasis. Antimicrob Agents Chemother 47: 2373-2375, 2003.

9. Kumamoto CA and Vinces MD: Contributions of hyphae and hypha-co-regulated genes to Candida albicans virulence. Cell Microbiol 7: 1546-1554, 2005.

10. Niewerth $M$ and Korting HC: Phospholipases of Candida albicans. Mycoses 44: 361-367, 2001.

11. Schaller M, Borelli C, Korting HC and Hube B: Hydrolytic enzymes as virulence factors of Candida albicans. Mycoses 48: 365-377, 2005.

12. Yang YL: Virulence factors of Candida species. J Microbiol Immunol Infect 36: 223-228, 2003.

13. Parker JC Jr, McCloskey JJ and Knauer KA: Pathobiologic features of human candidiasis. A common deep mycosis of the brain, heart and kidney in the altered host. Am J Clin Pathol 65: 991-1000, 1976.

14. Spellberg B, Ibrahim AS, Edwards JE Jr and Filler SG: Mice with disseminated candidiasis die of progressive sepsis. J Infect Dis 192: 336-343, 2005.

15. Schindler R, Krautzig S, Lufft V, et al: Induction of interleukin-1 and interleukin-1 receptor antagonist during contaminated in-vitro dialysis with whole blood. Nephrol Dial Transplant 11: 101-108, 1996.

16. Descamps-Latscha B, Herbelin A, Nguyen AT, et al: Balance between IL-1 beta, TNF-alpha, and their specific inhibitors in chronic renal failure and maintenance dialysis. Relationships with activation markers of T cells, B cells, and monocytes. J Immunol 154: 882-892, 1995.

17. O'Day DM, Head WS, Csank C, et al: Differences in virulence between two Candida albicans strains in experimental keratitis. Invest Ophthalmol Vis Sci 41: 1116-1121, 2000.

18. O'Day DM, Head WS, Robinson RD, Yang R, Shetlar D and Wang MX: Contact lens-induced infection - a new model of Candida albicans keratitis. Invest Ophthalmol Vis Sci 40: 1607-1611, 1999.

19. Odds FC, Brown AJ and Gow NA: Candida albicans genome sequence: a platform for genomics in the absence of genetics. Genome Biol 5: 230, 2004.

20. O'Day DM, Robinson R and Head WS: Efficacy of antifungal agents in the cornea. I. A comparative study. Invest Ophthalmol Vis Sci 24: 1098-1102, 1983.

21. Leunk RD and Moon RJ: Physiological and metabolic alterations accompanying systemic candidiasis in mice. Infect Immun 26: 1035-1041, 1979.

22. Hoyer LL, Clevenger J, Hecht JE, Ehrhart EJ and Poulet FM: Detection of Als proteins on the cell wall of Candida albicans in murine tissues. Infect Immun 67: 4251-4255, 1999. 